\title{
Former à la traduction multimodale
}

\section{Training students for multimodal translation}

\author{
Sophie Léchauguette \\ Univ. Lille, ULR 4074 - CECILLE - Centre d'Études en Civilisations Langues et Lettres \\ Etrangères, F-59000 Lille, France \\ sophie.lechauguette@univ-lille.fr
}

\begin{abstract}
Many books designed for international distribution combine text blocks and images. Their layout offers hybrid messages organized on the visual space of a double page. Texts both in the original language and in translation must fit into limited spaces or boxes positioned around illustrations. Thus, translators practice multimodal translation, writing texts that preserve or enhance the cohesion between visual and textual messages. This skill requires some training. Unfortunately, while theoretical writings on pragmatic translation acknowledge its intersemiotic nature, few training programs address this aspect. The creation of a course on multimodal translation would be a valued addition to any translator training program. The concept of a hybrid translation unit offers a way of structuring material to introduce both intersemiotic and multimodal translation in professional curricula. The author draws on her professional experience to discuss the role of illustrations in grasping meaning through practical examples. She suggests generalizable translation strategies to strengthen text-image cohesion, or even generate text from images alone, while adapting the book in translation to its intended readership.
\end{abstract}

Keywords: training, functionalism, intersemiotic translation, cohesion, hybrid translation unit

En termes d'action du traducteur, je conçois l'UT (unité de traduction) comme un balayage articulé qui part de la construction du sens (opération fondamentale ou de base) pour produire des équivalences (seconde phase de l'opération) visant à la réécriture d'un texte, dont la cohérence et l'acceptabilité vont générer un troisième type d'interventions de la part du traducteur, interventions qui souvent visent à restituer le liant du texte et ses qualités pragmatiques. 


\section{INTRODUCTION}

Les traducteurs de livres et documents illustrés réfléchissent sur un ensemble formé d'éléments textuels (rubriques, encadrés, légendes...) et visuels (photos, schémas, croquis, graphiques) organisés pour faciliter la compréhension des contenus informationnels. Dès 1971, Reiss signale qu'ignorer les illustrations revient à se priver de précieux compléments d'information : «En effet, les textes à traduire (présentés sous forme écrite) sont souvent intégrés dans un outil de communication plus vaste, incluant des éléments non verbaux tels que la gestuelle, les mimiques, les images, la musique. Ce supplément de complexité n'est bien sûr pas sans incidence sur la manière de traduire... et de critiquer les produits de traduction (116)».

Ainsi, une traduction négligeant l'entourage du texte est au mieux lacunaire et au pire erronée. Des sémioticiens pionniers comme Barthes (1964) et Eco (1968) ont ouvert la voie à l'analyse des productions multimodales : affiches et pages de publicité. L'analyse sémiotique permet de mieux saisir cet objet hybride qu'est le livre (ou tout document associant texte et image dans une maquette). Elle entre donc naturellement dans la réflexion traductologique. Suivant Torresi et Ketola, Tuominen et al. $(2018$, p. 3) regrettent la rareté des études sur la traduction multimodale en dehors des domaines techniques. Cet article apporte des éléments de réflexion pertinents dans tous les secteurs. Les apprentis traducteurs focalisent leur attention sur le texte mais négligent les illustrations. Analyser les images dans leur rapport au texte pour les intégrer à la réflexion traductive n'est pas une démarche spontanée. Qu'ils se destinent à la traduction dans le milieu de l'édition ou dans celui des agences de traduction, les jeunes traducteurs doivent apprendre à s'appuyer sur les illustrations pour comprendre, puis pour rédiger. L'ajout de la compétence multimodale sur le référentiel de compétences du réseau européen des masters en traduction pourrait inciter les équipes pédagogiques à systématiser l'utilisation de documents multimodaux comme support de cours. Beaucoup de professionnels apprennent à gérer les images en autodidacte. C'est regrettable. Les maladresses des novices témoignent de la nécessité d'un tel enseignement.

La première partie de cet article évoque des situations de traductions fréquentes révélatrices de l'utilité du recours aux illustrations - présentes, mal placées, voire absentes - pour traduire. La seconde rappelle les multiples rôles de l'image, et ses rapports au texte, variables selon ses fonctions communicatives. Partant d'analyse d'erreurs, la troisième partie propose des stratégies pédagogiques favorisant l'acquisition de ce savoir-faire nécessaire à la professionnalisation d'un traducteur de documents multimodaux. La démarche s'appuie sur la définition d'une unité de traduction hybride qui réunit éléments graphiques et langagiers dans la phase de construction du sens, préliminaire à la réécriture du texte. Le traducteur, au centre de la démarche, reconfigure cette unité à géométrie variable en associant des informations textuelles et visuelles, internes ou externes au document en traduction, au fur et à mesure de 
l'avancée du travail. C'est paradoxalement la rupture du lien logique entre texte et illustrations constatée dans des traductions de débutants qui fait ressortir la synergie existante entre ces deux modes de communication.

\section{ACCÈS À L'IMAGE}

Il faut savoir que les traducteurs ne disposent pas toujours du livre de départ, en cours de fabrication, ce qui les prive des illustrations associées au texte. Dans un marché du livre mondialisé, il n'est pas rare que des éditeurs décident de sortir simultanément un ouvrage dans plusieurs pays. Les délais de traduction étant très serrés, les traducteurs peuvent n'avoir que le texte, sans les illustrations, ou les recevoir à part dans un fichier pdf avant mise en page. Il arrive aussi de travailler à partir d'épreuves non définitives. S'inspirer des méthodes de la critique génétique, en s'intéressant aux documents de travail avant publication, apporte un éclairage sur les conditions de fabrication des ouvrages publiés et les vicissitudes méconnues qui conduisent les traducteurs à développer diverses stratégies indécelables à la critique fondée sur la comparaison d'originaux et de traduction publiée comme en témoignent les deux exemples suivants.

Renforcer la cohésion texte/image dans un document multimodal

Les traductrices d'un ouvrage de couture sur la modification des patrons, Pattern Cutting Primer disposaient d'une extraction du texte converti en word et des épreuves du livre avec les illustrations en cours de relecture en anglais au format pdf. La cohésion entre le texte et l'image est ici cruciale. Exemple 1: Lightly press the finished dart tuck. Any excess can be (1) pressed cleanly to one side or (2) divided equally either side of the stitch line.

Traduction exacte de la phrase de départ :

Presser légèrement la pince finie. Son volume peut être (1) soit couché d'un côté, (2) soit réparti symétriquement de part et d'autre de la ligne de piqûre

Au vu du schéma 3 p. 28, la correctrice demande l'inversion des propositions repérées ci-dessus par les chiffres entre parenthèses pour faire correspondre l'ordre dans lequel les deux positions décrites apparaissent dans la phrase à celui du schéma qui montre en premier, à gauche la répartition symétrique et en second, à droite, la pince couchée.

L'illustration commande une réorganisation syntaxique et l'inversion des informations pour le confort du lecteur. On peut donc lire :

«Son volume peut être soit (2) réparti symétriquement de part et d'autre de la ligne de piqûre, (1) soit couché d'un côté».

Exemple 2: La difficulté de traduction provenait moins de la technicité du sujet que d'une inversion des croquis destinés à expliquer la réalisation d'une poche avec 
un passepoil. Le texte et l'image des schémas 1 et 2 p. 169 des épreuves semblaient inversés. La traductrice, après avoir obtenu confirmation de la relectrice et conseillère technique, a pu rédiger une traduction cohérente et demander la remise en ordre des illustrations. Souvent les premiers à découvrir l'intégralité du livre, les traducteurs s'aperçoivent d'erreurs, moins rares qu'on ne le voudrait, et informent leur donneur d'ouvrage, assurant ainsi gracieusement une ultime correction du livre de départ. Quand l'avertissement arrive trop tard, certaines erreurs persistent parfois jusqu'à la publication. Deux photos, d'un pelham et d'un mors (objets en forme de H) sont à l'envers dans l'édition en anglais et dans le premier tirage de L'encyclopédie des chevaux, (Pickeral, 2003, p. 91). Il faudra attendre le deuxième tirage de la traduction française pour que l'éditeur pivote de $180^{\circ}$ la photo du mors, mais pas celle du pelham.

Besoin d'images extérieures au document en traduction

Les ouvrages illustrés ne sont pas les seuls à poser des problèmes d'accès à l'image. Traduire des textes très descriptifs, comme des guides de voyages au format de poche, qui comportent peu de photos, est une gageure. Faute d'information visuelle interne à l'ouvrage, le traducteur averti ressent le besoin de compléter l'information textuelle. La recherche d'images du référent, qui le donnent à voir, permet de trancher entre plusieurs interprétations possibles d'un même signifiant. Un adjectif comme " great » pour qualifier un escalier suggère la grandeur ; " magnifique, grandiose, extraordinaire » sont des traductions possibles, mais peu probables pour l'escalier d'une petite église de village en Bretagne. La démarche traductive demande une attention constante à la correspondance entre le texte et l'extra-linguistique. La vérification de l'absence de contradiction entre des termes corrects en tant que traduction du mot et le réel signifié passe souvent par la recherche d'un complément d'information graphique externe au livre en traduction. Les étudiants, qui passent souvent à côté des informations visuelles fournies, ne vont pas sans apprentissage compléter une information textuelle lacunaire par la recherche d'un complément visuel. Il faut développer cette compétence qui fait partie des stratégies documentaires de tout bon professionnel. La traduction d'ouvrages pragmatiques, souvent des manuels donnant des instructions, exige de veiller à la cohérence des deux modes de communication, voire à suppléer au manque si l'un des deux fait défaut dans le livre en traduction, pour donner une information exploitable au lecteur du livre traduit.

\section{ÉVOLUTION TECHNOLOGIQUE}

L'accès à l'image est aujourd'hui quasi immédiat mais avant internet et la dématérialisation des illustrations, la situation était plus difficile. Un éditeur d'art pouvait être dans l'impossibilité d'envoyer des illustrations, que lui-même attendait de recevoir. Il fallait alors « traduire en aveugle». Si les reproductions arrivaient chez 
l'éditeur, avant la remise de la traduction, un dialogue par téléphone entre traducteur et secrétaire d'édition permettait de convenir de la nuance d'un adjectif de couleur indécidable sans voir le référent. Une fleur dans une nature morte est-elle parme, lilas ou mauve ; une poterie, ocre, brun, marron ou bistre ? Quand les documents arrivaient trop tard, la décision finale échappait aux traducteurs. Ces difficultés anecdotiques font aujourd'hui sourire. Internet facilite l'accès à des reproductions nécessaires pour traduire les nuances, qu'elles figurent ou non dans le livre. Il faut toutefois se méfier des grandes variations dans le rendu des couleurs d'un écran à l'autre. Selon le degré de précision requis, la connaissance de l'œuvre originale reste la seule garante de l'exactitude de la traduction des couleurs.

Le tableau qui conclut cette première partie résume différentes situations de traduction qui échappent à la critique fondée sur la comparaison d'ouvrages publiés. Il souligne les opérations de traduction effectuée au niveau de la mise en relation du texte et de l'image au moment de la réalisation d'une traduction montrant l'intérêt d'une critique génétique des traductions multimodales.

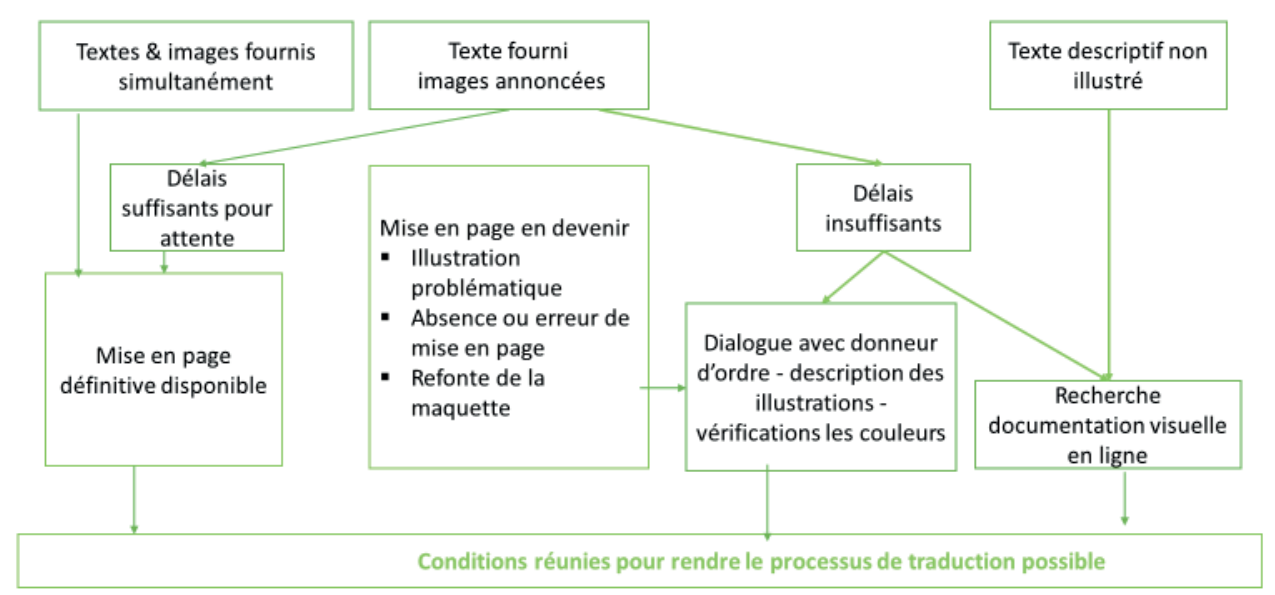

Fig. 1. Traduire des ouvrages illustrés: Quelques cas de figure issus de situations réelles

\section{TEXTES ET IMAGES DANS LES COLLECTIONS ILLUSTRÉES}

Les maquettes des ouvrages fournissent des informations pour penser la traduction multimodale. Une collection accueille des livres similaires dans une mise en page commune à tous les titres. Cette coquille vide offre un cadre interprétatif pour envisager la relation texte/image selon la visée des ouvrages. Le traducteur de métier y lit des instructions de traduction implicites que le formateur va s'employer à rendre visibles aux apprentis. Le statut relatif des illustrations par rapport aux textes cor- 
respond à des fonctions communicatives distinctes. Martinec et Salway (2005) ont élaboré une classification théorique très complète, trop complexe pour une utilisation pédagogique. Dans un souci de simplification, le formateur peut retenir les trois cas de figure les plus fréquents : l'image domine le texte, lui est subalterne ou complémentaire.

Un rapport image/texte à géométrie variable

L'université fait peu de cas des livres objets (coffee table books), destinés à être feuilletés, regardés, puis reposés. Le texte bref en regard des illustrations est anecdotique et sa fonction informative, réduite à la portion congrue. C'est un faire-valoir. La photo, œuvre ou représentation d'une œuvre, domine un texte souvent lénifiant à peine destiné à être lu. Au traducteur de fournir un texte qui remplira une fonction identique, même si l'adaptation culturelle requiert un message un peu différent. Dans un beau livre, le rapport entre les deux systèmes s'inverse. Sérieux et érudit, le texte sollicite un effort cognitif de la part des lecteurs. Les rubriques textuelles sont hiérarchisées. Le texte courant véhicule l'essentiel de l'information. Diverses rubriques viennent le compléter et accompagner les illustrations : légendes, vignettes, cartels, encadrés... Matérialisés par des polices de caractères, des fonds colorés, des cadres, ces rubriques forment une image visuelle cohérente, interprétable à l'échelle de la double page. Elles guident les lecteurs dans leurs recherches de différents types d'information. Souvent en italique, les légendes sautent aux yeux. Leur texte est plus rédigé que celui des cartels, quasiment sans syntaxe, qui se réduisent au nom de l'artiste, au titre et aux dimensions de l'œuvre ainsi qu'aux matériaux utilisés. Le texte courant reprend et développe ces informations dans un style défini par la charte éditoriale. Indépendamment des qualités ou faiblesses stylistiques du texte de départ, le texte d'arrivée privilégie l'agrément du lecteur. En effet, dans l'édition, la première consigne, tellement « évidente » qu'elle reste parfois dans le domaine du non-dit, est de produire une traduction plaisante à lire. Les chartes ou briefs de traduction fournissent des instructions précises aux auteurs et traducteurs. Elles préconisent ou proscrivent certaines tournures et imposent des termes ou choix orthographiques pour harmoniser les contenus textuels des ouvrages, renforçant par là même l'identité d'une collection au-delà du visuel créé par la maquette.

\section{Complémentarité de l'image}

Un exemple, dans le domaine de la cuisine, activité pratiquée par tous, illustre une problématique commune à celles de la plupart des guides pratiques et ouvrages consacrés à des artisanats d'art. Une photo du plat (ou projet) terminé, mis en valeur par une présentation esthétique, accompagne les recettes (ou explications). Il s'agit à la fois d'informer et d'inciter le lecteur à entreprendre la préparation ainsi illustrée. Un conflit entre la liste des ingrédients où figurent des lentilles vertes et la photo du plat qui montre des lentilles corail, deux variétés ayant des temps de cuisson diffé- 
rents, peut dissuader de préparer la recette. Un traducteur attentif corrige le problème en proposant une variante avec modification du temps de cuisson selon la variété choisie. Certaines collections de livres de cuisine et de loisirs créatifs privilégient le visuel en proposant une rubrique pas à pas. Une photo illustre les différentes étapes de la préparation, le texte se réduit à une instruction brève. Toujours présente, la fonction appellative passe au second plan derrière la fonction informative du langage, pour reprendre les catégories définies par Jakobson (1959). La valeur esthétique des illustrations les apparente aux affiches publicitaires, en particulier celles des pages de couverture destinées à attirer le regard du lecteur potentiel pour l'inciter à ouvrir le livre. L'œil parcourt la double page qui apparaît alors, passant des illustrations aux rubriques textuelles qui participent ensemble de la construction du sens.

Des lecteurs toutefois se contentent des illustrations et se dispensent de la lecture. L'absence d'ouvrages disponibles dans leur langue contraint certains lecteurs à acheter des ouvrages en langue étrangère. Leur besoin d'une traduction souligne l'absence d'autonomie de l'image qui ne se suffit pas à elle-même. Nous avons ainsi rencontré dans un groupe de sculpteurs sur bois, un lecteur francophone capable de se débrouiller sans le texte. Il avait assez d'expérience pour s'inspirer des modèles proposés sans lire les explications. En revanche, un tourneur sur bois, nous a demandé de traduire Multi-Axis Spindle Turning (Dill, 2018). Les explications techniques ne pouvaient être comprises en regardant les photos. Nous lui devons un magnifique support de cours pour une introduction à la traduction de textes spécialisés, pragmatiques et multimodaux. Cet ouvrage sur le tournage excentré digne de figurer au catalogue d'une collection comme Le geste et l'outil chez Eyrolles est l'occasion d'aborder la notion de collection, rarement évoquée en traductologie.

Traduire un ouvrage maquetté pour une collection

Une vignette à vocation commerciale annonce : « Le texte, technique et très précis, accompagne les progrès du lecteur. Il est soutenu par de nombreuses illustrations : photos couleurs et croquis (Quatrièmes de couverture, collection Le geste et l'outil) » Les traducteurs avertis y lisent une instruction de traduction : rédiger un texte précis et concis. Le contenu informatif est technique, mais le style est journalistique. C'est la différence entre traduction technique et pragmatique. Cette collection ne s'adresse pas à des lecteurs captifs, obligés de lire dans le cadre de leur métier ou parce qu'ils viennent d'acheter un outil dont ils « subissent » le manuel d'utilisation. Elle vise un public de lecteurs débutants et des amateurs avertis qui exigent des textes clairs et veulent une lecture gratifiante. Cette attente fait le succès des collections de manuels spécialisés rédigés dans une prose journalistique claire et illustrés de photos couleurs plus attrayantes que les croquis en noir et blanc des modes d'emploi traduits automatiquement avec des résultats discutables.

Le verbe soutenir suggère que l'image serait seconde par rapport au texte. Toutefois, sans illustration, certains passages seraient incompréhensibles et d'autres sem- 
bleraient incomplets. C'est bien sûr le cas de toutes les légendes : «Voici à quoi ressemble la bordure classique mise en valeur » (Moor, 2009, p. 60). Privés de la reproduction, les lecteurs de cet article ne sauront pas à quoi ressemble la bordure classique à moins d'aller voir dans le livre cité publié dans la collection Le geste et l'outil. La présence et le positionnement des illustrations dans la page influent sur la rédaction du texte courant. Les éditions Eyrolles ont refait la maquette du livre anglais jugé peu ergonomique puisque textes et illustrations pouvaient être distants de plusieurs pages. Il suffit parfois de placer une illustration en regard du texte pour le clarifier. L'élimination d'une photo se voulant humoristique, mais ridicule en traduction, a entraîné la réécriture de plusieurs passages renvoyant à ce trait d'humour (Léchauguette, 2011), ce qui est un exemple de reconfiguration d'une unité de traduction hybride en cours de traduction. L'intervention voulue par l'éditeur visait à restituer le « liant et la qualité du texte» (Ballard, 2006). Cette suppression était envisageable parce que le contrat entre les éditeurs prévoyait une refonte de la maquette, cas moins fréquent que le contrat de coédition.

\section{Le contrat de coédition}

En cas de coédition, la maquette devient un élément visuel invariant. Le seul moyen de modifier une illustration est d'incruster du texte sur l'image, ou de la barrer d'une croix. Si pour diverses raisons d'ordre culturel, la présence d'une image demande une adaptation, celle-ci peut se faire dans le texte courant, en ajoutant une légende ou un commentaire. Par ses remarques critiques et ses suggestions, le traducteur, souvent découvreur de l'intégralité du texte, contribue à renforcer la cohésion linguistico-visuelle du message. La traduction contribue alors à l'amélioration du livre. Rares sont les traducteurs formés à cette compétence visuelle, comme le déplore Damaskinidis (2016) appelant de ses vœux la systématisation d'une formation répondant à ce besoin (316). Comme cet auteur, et sans doute d'autres, nous sensibilisons déjà nos étudiants à la présence des illustrations et les incitons à en exploiter tout le potentiel documentaire.

\section{DES ACTIVITÉS PÉDAGOGIQUES POUR INTÉGRER L'IMAGE À LA RÉFLEXION TRADUCTIVE}

Ainsi, la première partie d'un examen donné en fin de semestre d'initiation à la traduction dans le domaine médical consistait à traduire une explication en six étapes, légendes et dessins, montrant comment se laver les mains. En pleine épidémie de COVID, ce type d'affiche faisait partie de notre quotidien. L'énoncé précisait de veiller à la cohérence entre le texte et l'image puisque l'instruction sous la deuxième image ne correspondait pas. Le tiers des étudiants a proposé une instruction cohérente, rédigée d'après l'image, qui soulignait l'importance du lavage du dos de 
la main, qui sans cette instruction était oublié. Une étudiante a réécrit la légende et profité de la possibilité donnée de « communiquer avec le donneur d'ordre » lui écrivant : «Remarque : la figure 2 ne montre pas un lavage paume contre paume comme indiqué dans le texte anglais mais la paume d'une main contre le dos de l'autre » témoignant par cette remarque de son savoir-faire et de son savoir être. Cependant, tous ont traduit « rub » par « frotter », personne n'utilisant « savonner » pourtant suggéré par les bulles en arrière-plan, terme simple et utile pour varier le lexique et éviter des répétitions.

Une progression pédagogique pourrait commencer par donner à traduire des schémas sans texte, puis des documents dont les illustrations aident à comprendre le texte, avant de présenter des illustrations sources de contraintes et enfin des illustrations en conflit avec le texte. Bien guidés, les étudiants apprennent à décrypter les consignes implicites contenues dans la maquette même d'un document. Les premiers chapitres d'ouvrages d'initiation à des métiers d'art présentent sous forme textuelle et visuelle les outils et les matières travaillées. Les outils les plus spécialisés, absents des dictionnaires généralistes, sont inconnus des étudiants comme des traducteurs expérimentés ou des lecteurs désireux de s'initier à une pratique. Tous découvrent le lexique technique dans les premières pages et souvent dans les deux langues. Faute de dictionnaires ou de corpus, la recherche documentaire lexicale s'appuie sur le visuel. Les catalogues illustrés de fournisseurs constituent d'excellentes sources de documentation visuelle, en particulier quand ils existent en plusieurs langues.

\section{Comportement traductif des novices}

En France, les apprentis traducteurs issus des premiers cycles de langue ont une approche de la traduction très littérale centrée sur le respect de textes de départ rarement illustrés. Et s'ils le sont, l'image ne sera pas traitée dans le cours. Intégrer l'iconographie à la réflexion demande de modifier des habitudes de pensée inculquées par la formation initiale. Proposer des traductions intersémiotiques, par exemple faire rédiger des consignes pour des schémas de montage ou des explications strictement visuelles peut avoir un effet salutaire et convaincre l'étudiant qu'un bon traducteur est un rédacteur. Le texte peut ensuite revenir dans un environnement multimodal. L'exemple suivant reproduit deux phrases (pas des légendes) consécutives dans un texte courant mais séparées par une photo.

Phrase 1 : This image clearly shows the axis used between centers and the completed first profile (Dill, 2018, p. 27).

Une étudiante traduit: Sur cette image, on voit très bien l'axe entre-pointes et le premier profil.

La désignation de l'illustration par un calque est un premier problème, le second relève de la syntaxe. Au niveau lexical, les termes «photo » ou « illustration » sont préférables. Au niveau syntaxique, les consignes éditoriales préconisent d'utiliser ces termes en sujet : 
«Cette photo montre très bien le premier axe entre-pointes et le premier profil terminé »

Cette traduction, qui renforce la cohésion entre les deux systèmes de signification, est meilleure.

Phrase 2 : Now the spindle is moved to the second axis.

La photo montre l'ébauche déjà positionnée, prête à être tournée sur le second axe et non le passage du premier au second axe. La légende est exacte en anglais, mais sa traduction en français « On place maintenant l'ébauche prête pour être tournée sur le deuxième axe » est partiellement inexacte. Une réécriture s'impose pour « restituer le liant » entre le texte et l'image et renforcer les qualités pragmatiques de l'ensemble. Mieux vaut donc s'inspirer du visuel pour écrire par exemple : [Après avoir été tournée sur le premier axe choisi] l'ébauche est maintenant positionnée pour (ou prête à) être tournée sur le deuxième axe. Le segment entre crochets renforce la cohésion de l'unité de traduction multimodale où texte et illustrations participent à égalité dans la construction du sens formant une continuité visuelle.

Contrairement aux idées reçues, et comme en témoigne l'exemple de la traduction d'une affichette sur l'hygiène des mains, la plus grande difficulté n'est pas toujours la technicité. Des sujets familiers posent aussi de redoutables problèmes. Les apprenants négligeant le potentiel documentaire des illustrations écrivent des phrases qui prêtent à sourire. Ainsi pour traduire « Horses in the wild do not need rugs. Their coats grow thick and long in winter to protect them from the cold (Fitzpatrick, 2003, p. 380) ». La deuxième phrase établit la fonction de l'objet « rug »: protéger du froid ; la photo montre un cheval avec une couverture. Négligeant ces informations textuelles (contexte) et visuelles, (co-texte), un étudiant traduit : " Les chevaux sauvages n'ont pas besoin de tapis de selle ». Cette proposition suggère une réflexion exclusivement linguistique ignorant le réel. La recherche de colocations possibles donne « tapis de selle », objet qui se place sous une selle. Il suffit de chercher une image pour valider ou non le résultat de sa recherche lexicale. Révélatrice de l'erreur, l'absence de correspondance entre le mot et l'image invite à poursuivre la recherche. Négligeant aussi l'apport des images, année après année, d'autres écrivent « tuteur », terme impropre, à la place de «montant» dans la traduction d'un extrait sur la vannerie. Cette erreur récurrente est pourtant facile à éviter car les schémas légendés sont facilement accessibles en ligne et présents dans tous les ouvrages sur le sujet. Encore faut-il avoir appris à aller chercher des illustrations.

La traduction multimodale, qui incite à nommer l'objet représenté plutôt qu'à chercher la traduction d'un terme, s'inscrit dans le processus de déverbalisation préconisé par l'école de Paris. 


\section{CONCLUSION}

Les difficultés constatées chez des étudiants découvrant la traduction d'ouvrages illustrés plaident pour l'intégration d'une formation à la traduction multimodale dans leur cursus. Et cette formation doit pouvoir s'appuyer sur une recherche théorique sur la traduction intersémiotique qu'appellent de leurs vœux, à la suite de Reiss, des chercheurs comme Tomaszkiewicz et Froeliger :

Il est donc largement temps de revenir sur l'idée de Jakobson et d'intégrer dans la recherche traductologique la traduction intersémiotique, jusqu'à présent ignorée ou sous-estimée, tant au niveau pratique que théorique (Tomaszkiewicz, 2005, p. 167).

«D'où l'importance, trop souvent occultée par ceux qui n'ont de ces textes qu'une vision linguistique, des aspects non verbaux : la traduction est une affaire intersémiotique » (Froeliger, 2013, p. 39).

Une réflexion pédagogique ancrée dans la pratique du métier montre l'urgence de systématiser l'offre de cours de traduction intersémiotique et multimodale dans la formation des professionnels. Livres et documents divers demandent d'apprendre à analyser et utiliser les images internes, celles qui accompagnent le texte, et d'ap-prendre à recourir à des images externes dans le cadre de la recherche documentaire et lexicologique. En sollicitant les traducteurs formateurs dans leurs retranchements, la pédagogie nourrit la réflexion théorique et fait émerger nombre de paramètres invisibles aux critiques disposant des seuls documents originaux et de leur traduction. Les différences observées ne permettent pas d'en saisir les motivations profondes exigées par la nature multimodale de l'offre d'information. Les traducteurs-formateurs occupent une position charnière d'où ils enrichissent et puisent dans la réflexion théorique par leur pratique de la traduction multimodale et de son enseignement. 


\section{BIBLIOGRAPHIE}

Ballard, M. (2006). À propos des procédés de traduction. Palimpsestes, 113-130. Retrieved from : https:// doi.org/10.4000/palimpsestes.386.

Barthes, R. (1964). Rhétorique de l'image. Communications, 4, 40-51.

Damaskinidis, G. (2016). The Visual Aspect of Translation Training in Multimodal Texts. Meta, 61, 2, 251-500, doi.org/10.7202/1037761ar.

Eco, U. (1968). La structure absente (trad. Uccio Esposito-Torrigiani). Paris : Mercure de France, 1972, La struttura assente, Milano : Valentino Bompiani, 253-256.

Froeliger, N. (2013). Les Noces de l'analogique et du numérique : de la traduction pragmatique. Paris : Les Belles Lettres.

Jakobson, R. (1959). Les Aspects linguistiques de la traduction. Essai de Linguistique Générale. Paris : Mouton, 260-266.

Ketola, A. (2017). Using translation research to model word image interaction. New Voices in Translations Studies, 82-104.

Léchauguette, S. (2011). Les stratégies de l'humour dans les textes pragmatiques. Humoresque, 34, 147 164.

Martinec, R. \& Salway, A. (2005). A system for image-text relations in new (and old) media. Visual Communication, 4 (3), 339-374.

Reiss, K. (2009). Problématiques de la traduction (trad. Catherine Bocquet). Paris : Economica - Anthropos (original work published 1995).

Tomaszkiewicz, T. (2005). La traduction intersémiotique fait-elle partie de la traductologie ? In J. Peeters (ed.), La traduction de la Théorie à la pratique et retour (pp. 159-168). Rennes : Presses Universitaires de Rennes.

Tuominen, T., Hurtado, C.-J. \& Ketola, A. (2018). Why methods matter: Approaching multimodality in translation research. Linguistica Antverpiensia. New Series: Themes in Translation Studies, 17, 1-21.

\section{CORPUS}

Barnfield, J. (2012). Pattern Making Primer. Londres : Bloomsbury, 28, 169. Dill, B. (2018). Multi-Axis Spindle Turning, Atglen : Schiffer Publishing, 27.

Fitzpatrick, A. (2003). The Ultimate Guide to Horsebreeds, Edison : Chartwell Books Inc, 380.

Moor, D. (2009). Sculpture au couteau, col. Le geste et l'outil. Paris : Eyrolles.

Pickeral, T. (2003). L'encyclopédie des chevaux. Paris : Parragon, 91. 